\title{
Synthesis of spiro-linked macrocyclic crown formazans and a bis(crown formazan)
}

\author{
Ashraf A. Abbas and Ahmed H. M. Elwahy* \\ Chemistry Department, Faculty of Science, Cairo University, Giza, Egypt \\ E-mail: aelwahy@hotmail.com
}

\begin{abstract}
The spiro-linked crown formazans 7a,b and bis(crown formazan) 11 were synthesized by diazotization of tetrakis(amine) hydrochlorides $\mathbf{6}$, and 10, respectively, followed by coupling with malonic acid or cyanoacetic acid in pyridine.
\end{abstract}

Keywords: Alkylation, diazotization, coupling, macrocyclic formazan

\section{Introduction}

The chemistry and applications of acyclic formazans as well as monocyclic crown-formazans have received much attention and are the subject of several reviews. ${ }^{1}$ Many macrocyclic formazans have been synthesized, and their applications in selective metal extraction ${ }^{2}$ and determination $^{3}$ have been studied. Such applications depend mainly on the cavity size of the macrocyclic crown formazans as well as on the substituents on the macrocycle. Recently, interest has been directed toward the synthesis of multi-site crown compounds. These multi-site molecular receptors are capable of binding two or more guest metal cations. ${ }^{4}$

Previously, we have reported the synthesis of macrocyclic formazans $\mathbf{1}$ by coupling of the appropriate bis-diazonium salt with active methylene compounds under high dilution conditions; we have studied their behavior in the spectrophotometric determination of lithium and as carriers in cesium ion selective electrodes. ${ }^{1 \mathrm{c}, 5 \mathrm{a}-\mathrm{f}}$

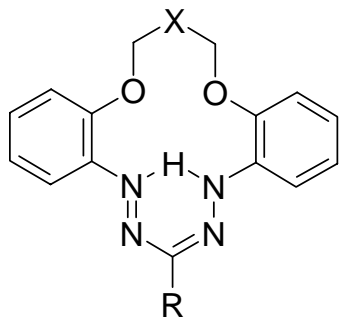

1

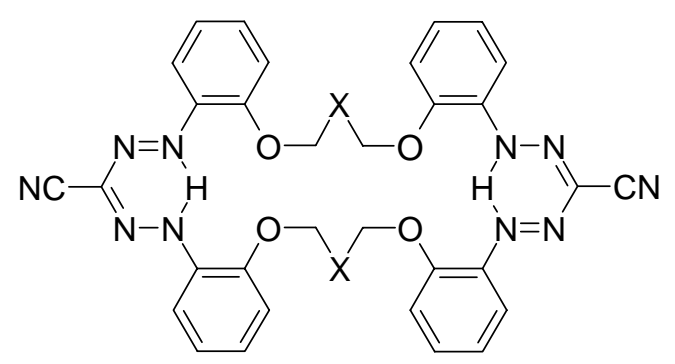

2 
In order to improve the cation binding abilities of macrocycles $\mathbf{1}$, Katritzky et al. ${ }^{\text {lb }}$ have reported the synthesis of lariat crown-formazan 1 ( $\mathrm{X}=\mathrm{CHO}(\mathrm{C}=\mathrm{O}) \mathrm{CH}_{2} \mathrm{NMe}_{2}, \mathrm{R}=\mathrm{Ph}$ ) containing a strong donor group as a supporting ligand at the end of the side arm. Moreover, $\mathrm{Abbas}^{5 \mathrm{f}}$ reported the synthesis of crown formazans 2 with two formazyl moieties.

We report here on the first synthesis of spiro-linked crown formazans 7a,b and that of bis(crown formazan) 11, which are expected to be analytically useful on account of their ability to bind more than one metal ion.

\section{Results and Discussion}

For the synthesis of compounds 7a,b, first the tetrakis(acetamido) derivative 5 was prepared by the reaction of the potassium salt of $o$-acetamidophenol (3) with pentaerythrityl tetrabromide (4) in boiling DMF (Scheme 1). Compound 5 was hydrolyzed with an ethanolic solution of hydrochloric acid to give the tetrakis(amine) hydrochloride $\mathbf{6}$. Diazotization of $\mathbf{6}$ with sodium nitrite in hydrochloric acid followed by coupling with malonic acid or cyanoacetic acid in pyridine yielded the corresponding macrocyclic formazans $7 \mathbf{a}, \mathbf{b}$ as deep red crystals (16\% and 18\%).

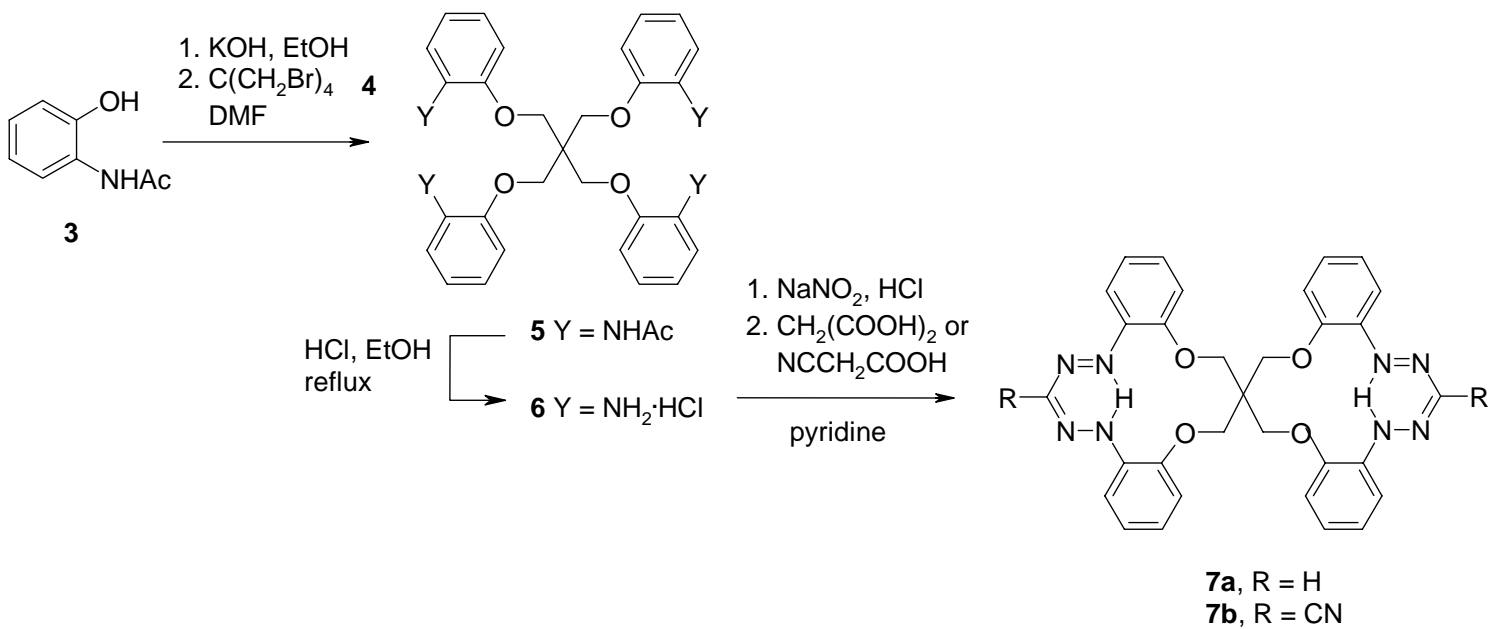

\section{Scheme 1}

Substitution of tetrakis(bromomethyl)benzene (8) with four equivalents of the potassium salt of $o$-acetamidophenol (3) in refluxing DMF gave the corresponding tetrakis(acetamidophenoxymethyl)benzene 9 as colorless crystals (92\%; Scheme 2). Treatment of the latter with an ethanolic solution of hydrochloric acid gave the tetrakis(amine) hydrochloride 10 (88\%). Diazotization of $\mathbf{1 0}$ with sodium nitrite in hydrochloric acid followed by coupling with malonic acid in pyridine and subsequent purification gave $\mathbf{1 1}$ as deep red crystals (15\%). No traces of the isomeric bis(crown formazan) $\mathbf{1 2}$ has been obtained from the coupling reaction. 


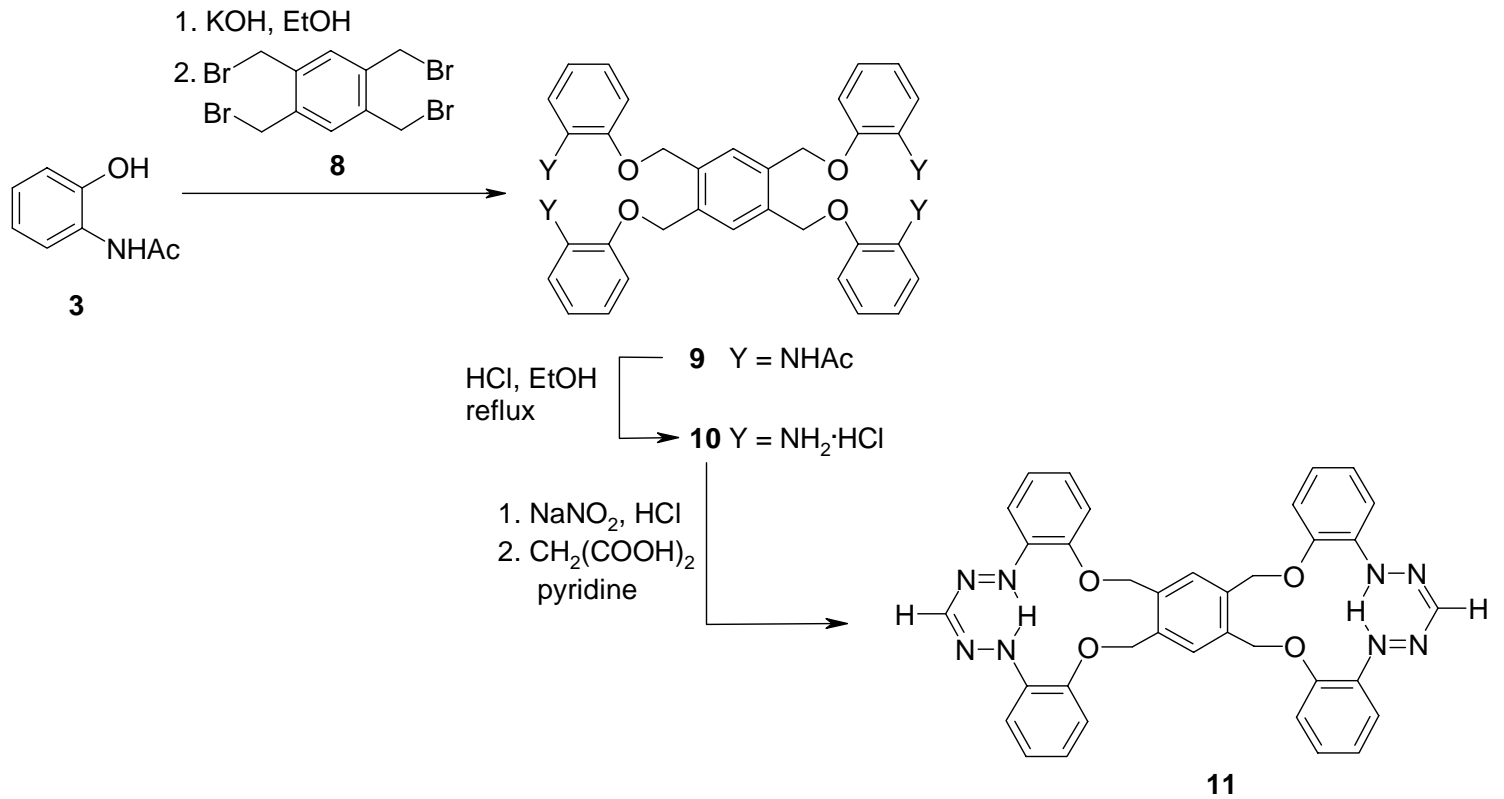

\section{Scheme 2}

The structure of compound $\mathbf{1 1}$ was confirmed by comparison with related crown formazans $2^{5 \mathrm{f}}, 13^{5 \mathrm{c}}, 14^{4 \mathrm{~b}}$.

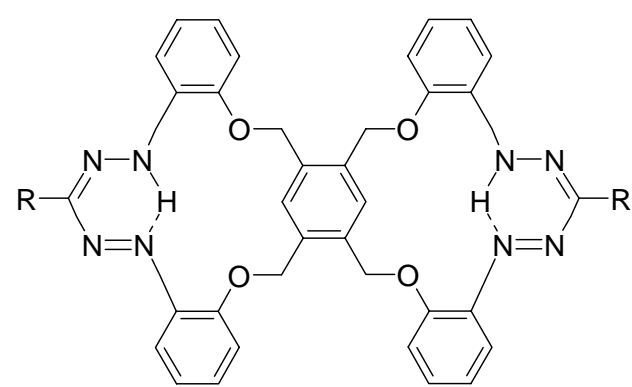

12

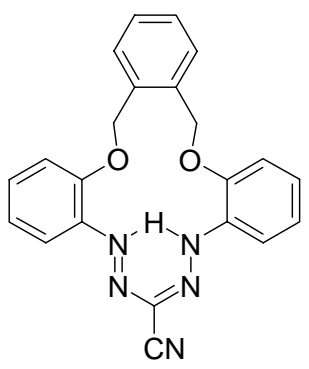

13<smiles></smiles>

14

Comparison of the positions of the $\mathrm{NH}$ signals in the ${ }^{1} \mathrm{H}$ NMR spectra of the proposed structure 11 with those of crown formazans $\mathbf{1 3}$ and $\mathbf{1 4}$ as well as macrocyclic bis(formazans) 2 led to the assumption of the formation of $\mathbf{1 1}$ rather than 12. Compounds $\mathbf{1 1}$ and $\mathbf{1 3}$ exhibit sharp $\mathrm{NH}$ signals in the downfield region at $\delta 15.70$ and 15.43, respectively, indicative of an intramolecular hydrogen bond. ${ }^{6}$

On the other hand, the NH signal of the macrocyclic formazan 14 was found at $\delta 11.94$, for 2 at $\delta$ 10.73-13.25. These upfield shifts of the NH signal indicates a weakening of the intramolecular hydrogen bond, which can be attributed to the increased size of the macrocycles 2 and 14 compared with that of 13 . The absence of a characteristic NH signal in the region $\delta 10-13$ of the product molecule supports the supposed structure 11. 
Structures of 7a,b, 11 were confirmed by IR, NMR and MS and elemental analyses. The symmetry of the novel compounds is manifested by a singlet characteristic of the four equivalent $\mathrm{OCH}_{2}$ groups in the ${ }^{1} \mathrm{H}$ NMR spectrum.

\section{Experimental Section}

General Procedures. IR spectra were recorded on a Perkin-Elmer 1430 spectrophotometer. NMR spectra were measured with a Varian Mercury $300\left({ }^{1} \mathrm{H}: 300 \mathrm{MHz},{ }^{13} \mathrm{C}: 75 \mathrm{MHz}\right)$. Mass spectra were recorded on GC MS-QP1000 EX (70 eV) or MS $5988(15 \mathrm{eV})$. Elemental analyses were carried out at the Microanalytical Centre, Cairo University.

Pentaerythrityl tetrabromide and tetrakis(bromomethyl)benzene were used as purchased from Aldrich.

Tetrakis(2-aminophenoxymethyl)methane tetrahydrochloride (6). o-Actamidophenol (3) (3.02 g, $20 \mathrm{mmol}$ ) was dissolved in hot ethanolic $\mathrm{KOH}$, prepared by dissolving $\mathrm{KOH}$ (1.12 g, 20 $\mathrm{mmol})$ in absolute ethanol $(20 \mathrm{~mL})$. The solvent was then removed in vacuo; the residual material was dissolved in DMF (15 mL), and pentaerythrityl tetrabromide (4) (1.94 g, $5 \mathrm{mmol}$ ) was added. The reaction mixture was refluxed for $15 \mathrm{~min}$, and $\mathrm{KBr}$ precipitated. $\mathrm{KBr}$ was filtered off, and the solvent was removed in vacuo. The residue, tetrakis(2-acetamidophenoxymethyl)methane (5) without further purifcation was used for the next step.

To the solution of 5 in absolute ethanol $(20 \mathrm{~mL})$ was added concentrated hydrochloric acid (16 $\mathrm{mL}$ ). The reaction mixture was then heated under reflux for $2 \mathrm{~h}$. After the solvent was removed in vacuo, the remaining solid was collected and crystallized from ethanol/ $\mathrm{HCl}$ to give colorless crystals $6(2 \mathrm{~g}, 62 \%) ; \mathrm{mp}>310^{\circ} \mathrm{C}$. IR $(\mathrm{KBr}): \tilde{v} 3417(\mathrm{NH}) \mathrm{cm}^{-1} .{ }^{1} \mathrm{H}$ NMR $\left(300 \mathrm{MHz}, \mathrm{D}_{2} \mathrm{O}\right): \delta$ $4.79\left(\mathrm{~s}, 8 \mathrm{H}, 4 \mathrm{OCH}_{2}\right), 7.15-7.57\left(\mathrm{~m}, 16 \mathrm{H}, \mathrm{H}_{\mathrm{Ar}}\right)$. Anal. calcd. for $\mathrm{C}_{29} \mathrm{H}_{36} \mathrm{Cl}_{4} \mathrm{~N}_{4} \mathrm{O}_{4}$ (646.44): C, 53.88; H, 5.61; N, 8.67. Found: C, 54.02; H, 5.85; N, 8.48.

1,2,4,5-Tetrakis(2-acetamidophenoxymethyl)benzene (9) and 1,2,4,5-tetrakis(2-aminophenoxymethyl)benzene tetrahydrochlorides (10). o-Acetamidophenol (3) (3.02 g, $20 \mathrm{mmol}$ ) was dissolved in hot ethanolic $\mathrm{KOH}$, prepared by dissolving $(1.12 \mathrm{~g}, 20 \mathrm{mmol})$ in absolute ethanol $(20 \mathrm{~mL})$. The solvent was then removed in vacuo; the residual material was dissolved in DMF (15 mL), and tetrakis(bromomethyl)benzene (8) (2.25 g, $5 \mathrm{mmol}$ ) was added. The reaction mixture was refluxed for $15 \mathrm{~min}$, and $\mathrm{KBr}$ precipitated. $\mathrm{KBr}$ was filtered off, and the solvent was removed in vacuo. The remaining solid was collected and crystallized from ethanol to give colorless crystals 9 (3.36 g, 92\%); mp 218-220 ${ }^{\circ} \mathrm{C}$. IR (KBr): $\tilde{v} 3282(\mathrm{NH}), 1697(\mathrm{CO}) \mathrm{cm}^{-1} .{ }^{1} \mathrm{H}$ NMR (300 MHz, $\left.\mathrm{CDCl}_{3}\right): \delta 1.96\left(\mathrm{~s}, 12 \mathrm{H}, 4 \mathrm{CH}_{3}\right), 5.21\left(\mathrm{~s}, 8 \mathrm{H}, 4 \mathrm{OCH}_{2}\right), 6.91-8.27\left(\mathrm{~m}, 22 \mathrm{H}, \mathrm{H}_{\mathrm{Ar}}\right.$, $4 \mathrm{NH}) .{ }^{13} \mathrm{C} \mathrm{NMR}\left(\mathrm{CDCl}_{3}, 75 \mathrm{MHz}\right): \delta 24.6\left(\mathrm{CH}_{3}\right), 70.0\left(\mathrm{OCH}_{2}\right), 113.55,121.47,122.91,124.27$, 128.93, 131.78, 135.76, $146.79\left(\mathrm{C}_{\mathrm{Ar}}\right), 168.60(\mathrm{C}=\mathrm{O})$. Anal. calcd. for $\mathrm{C}_{42} \mathrm{H}_{42} \mathrm{~N}_{4} \mathrm{O}_{8}(730.82)$ : $\mathrm{C}$, 69.03; H, 5.79; N, 7.67. Found: C, 68.98; H, 5.60; N, 7.79. 
To a solution of 9 (3.65 g, $5 \mathrm{mmol}$ ) in absolute ethanol $(20 \mathrm{~mL})$ was added concentrated hydrochloric acid $(16 \mathrm{~mL})$. The reaction mixture was then heated under reflux for $2 \mathrm{~h}$. After the solvent was removed in vacuo, the remaining solid was collected and crystallized from ethanol/ $\mathrm{HCl}$ to give colorless crystals $10(3.11 \mathrm{~g}, 88 \%) ; \mathrm{mp}>360{ }^{\circ} \mathrm{C}$. IR $(\mathrm{KBr}): \tilde{v} 3417(\mathrm{NH})$ $\mathrm{cm}^{-1} .{ }^{1} \mathrm{H}$ NMR $\left(300 \mathrm{MHz}, \mathrm{D}_{2} \mathrm{O}\right): \delta 5.55\left(\mathrm{~s}, 8 \mathrm{H}, 4 \mathrm{OCH}_{2}\right), 7.10-7.48\left(\mathrm{~m}, 16 \mathrm{H}, \mathrm{H}_{\mathrm{Ar}}\right), 7.79(\mathrm{~s}, 2 \mathrm{H}$, $\mathrm{H}_{\mathrm{Ar}}$ ). Anal. calcd. for $\mathrm{C}_{34} \mathrm{H}_{38} \mathrm{Cl}_{4} \mathrm{~N}_{4} \mathrm{O}_{4}$ (708.52): C, 57.64; H, 5.41; N, 7.91. Found: C, 57.33; H, $5.75 ; \mathrm{N}, 8.03$.

\section{Formazans 7a,b and 11. General procedure}

A solution of the appropriate tetrakis(amine hydrochlorides) 6, 10 (1 mmol) in water $(5 \mathrm{~mL})$ and conc. $\mathrm{HCl}(3 \mathrm{~mL})$ was diazotized at $-5{ }^{\circ} \mathrm{C}$ with a solution of sodium nitrite $(0.23 \mathrm{~g}$ in $5 \mathrm{~mL}$ water) for $0.5 \mathrm{~h}$. Stirring was continued for $1 \mathrm{~h}$ at $-5{ }^{\circ} \mathrm{C}$, and then the reaction mixture was added dropwise to a stirred solution containing malonic acid or cyanoacetic acid $(2 \mathrm{mmol})$ in pyridine $(150 \mathrm{~mL}), \mathrm{CuSO}_{4} .5 \mathrm{H}_{2} \mathrm{O}(0.5 \mathrm{~g})$, and water $(20 \mathrm{~mL})$ over a period of $1 \mathrm{~h}$. The reaction mixture was stored at $0{ }^{\circ} \mathrm{C}$ overnight. Upon addition of concentrated hydrochloric acid the solid precipitate formed was collected and purified by preparative TLC using silica gel $\left(60 \mathrm{~F}_{254}\right)$ with proper eluents.

Macrocyclic formazan 7a. Following the general procedure, $6(0.65 \mathrm{~g}, 1 \mathrm{mmol})$ and malonic acid $(0.21 \mathrm{~g}, 2 \mathrm{mmol})$ gave after purification using $\mathrm{CH}_{2} \mathrm{Cl}_{2}$ as an eluent $\left(\mathrm{R}_{f}=0.65\right)$ deep red crystals 7a (92 mg, 16\%); mp 272-274 ${ }^{\circ} \mathrm{C}$. IR (KBr): $\tilde{v} 3428(\mathrm{NH}) \mathrm{cm}^{-1}$. ${ }^{1} \mathrm{H}$ NMR $(300 \mathrm{MHz}$, $\left.\mathrm{CDCl}_{3}\right): \delta 4.51\left(\mathrm{~s}, 8 \mathrm{H}, 4 \mathrm{OCH}_{2}\right), 6.92-7.85\left(\mathrm{~m}, 16 \mathrm{H}, \mathrm{H}_{\mathrm{Ar}}\right), 8.36(\mathrm{~s}, 2 \mathrm{H}, 2$ Formazyl-CH), 15.76 (s, 2H, 2 NH, $\mathrm{D}_{2} \mathrm{O}$ exchangeable). MS: m/z (\%) 576 (100, $\mathrm{M}^{+}$), 533 (18), 307 (11), 145 (29). Anal. calcd. for $\mathrm{C}_{31} \mathrm{H}_{28} \mathrm{~N}_{8} \mathrm{O}_{4}$ (576.62): C, 64.57; H, 4.89; N, 19.43. Found: C, 64.93; H, 4.85; N, 19.30 .

Macrocyclic formazan 7b. Applying the general procedure, $6(0.65 \mathrm{~g}, 1 \mathrm{mmol})$ and cyanoacetic acid $(0.17 \mathrm{~g}, 2 \mathrm{mmol})$ gave after purification using $\mathrm{CH}_{2} \mathrm{Cl}_{2}$ as an eluent $\left(\mathrm{R}_{f}=0.48\right)$ deep red crystals $7 \mathbf{b}(112 \mathrm{mg}, 18 \%)$; mp 236-238 ${ }^{\circ} \mathrm{C}$. IR (KBr): $\tilde{v} 3383(\mathrm{NH}), 2229(\mathrm{CN}) \mathrm{cm}^{-1}$. ${ }^{1} \mathrm{H}$ NMR $\left(300 \mathrm{MHz}, \mathrm{CDCl}_{3}\right): \delta 4.44\left(\mathrm{~s}, 8 \mathrm{H}, 4 \mathrm{OCH}_{2}\right), 7.06-7.93\left(\mathrm{~m}, 16 \mathrm{H}, \mathrm{H}_{\mathrm{Ar}}\right), 16.03\left(\mathrm{~s}, 2 \mathrm{H}, 2 \mathrm{NH}, \mathrm{D}_{2} \mathrm{O}\right.$ exchangeable). MS: m/z (\%) 626 (35.7, $\left.\mathrm{M}^{+}\right), 570$ (39), 507 (61), 386 (30), 120 (100). Anal. calcd. for $\mathrm{C}_{33} \mathrm{H}_{26} \mathrm{~N}_{10} \mathrm{O}_{4}$ (626.64): C, 63.25; H, 4.18; N, 22.35. Found: C, 63.51; H, 4.30; N, 22.20. Macrocyclic formazan 11. Applying the general procedure, $10(0.71 \mathrm{~g}, 1 \mathrm{mmol})$ and malonic acid $(0.21 \mathrm{~g}, 2 \mathrm{mmol})$ gave after purification using $\mathrm{CH}_{2} \mathrm{Cl}_{2} /$ petroleum ether $\left(40-60{ }^{\circ} \mathrm{C}\right)(5: 1)$ as eluent $\left(\mathrm{R}_{f}=0.45\right)$ deep red crystals $11(95.7 \mathrm{mg}, 15 \%) ; \mathrm{mp}>360{ }^{\circ} \mathrm{C}$. IR $(\mathrm{KBr}): \tilde{v} 3428(\mathrm{NH})$ $\mathrm{cm}^{-1} .{ }^{1} \mathrm{H}$ NMR $\left(300 \mathrm{MHz}, \mathrm{DMSO}-d_{6}\right): \delta 5.42\left(\mathrm{~s}, 8 \mathrm{H}, 4 \mathrm{OCH}_{2}\right), 7.01\left(\mathrm{t}, J=7.5 \mathrm{~Hz}, 4 \mathrm{H}, \mathrm{H}_{\mathrm{Ar}}\right)$, $7.33\left(\mathrm{t}, J=7.5 \mathrm{~Hz}, 4 \mathrm{H}, \mathrm{H}_{\mathrm{Ar}}\right), 7.58-7.65\left(\mathrm{~m}, 8 \mathrm{H}, \mathrm{H}_{\mathrm{Ar}}\right), 7.87\left(\mathrm{~s}, 2 \mathrm{H}, \mathrm{H}_{\mathrm{Ar}}\right), 8.25(\mathrm{~s}, 2 \mathrm{H}, 2$ Formazyl$\mathrm{CH}), 15.43$ (s, 2H, 2 NH, $\mathrm{D}_{2} \mathrm{O}$ exchangeable). MS: $m / z$ (\%) $638\left(6.3, \mathrm{M}^{+}\right), 530$ (4), 354 (13), 183 (17), 109 (100). Anal. calcd. for $\mathrm{C}_{36} \mathrm{H}_{30} \mathrm{~N}_{8} \mathrm{O}_{4}$ (638.69): C, 67.70; H, 4.73; N, 17.54. Found: C, 67.30; H, 5.06; N, 17.40. 


\section{References}

1. (a) Ibrahim, Y. A.; Abbas, A. A.; Elwahy, A. H. M. J. Heterocycl. Chem. 2004, 41, 135. (b) Katritzky, A. R.; Belyakov, S. A.; Durst, H. D. Tetrahedron Lett. 1994, 35, 6465. (c) Ibrahim, Y. A.; Elwahy, A. H. M.; Abbas, A. A. Tetrahedron 1994, 50, 11489.

2. (a) Niz'eva, N. V.; Ionov, P. V.; Pletnev, I. V.; Kumina, D. M.; Ostrovskaya, V. M.; D'yakonova, I. A.; Zolotov, Yu. A. Dokl. Akad. Nauk SSSR, 1984, 274, 611-615. Chem. Abstr. 1984, 101, 47705. (b) Zolotov, Yu. A.; Ionov, V. P.; Bodnyav, A. V.; Larikova, G. A.; Niz' eva, N. V.; Vlasova, G. E.; Rybakova, E. V. Zr. Anal. Khim. 1982, 37, 1543. Chem. Abstr. 1983, 98, 78972. (c) Isakova, N. V.; Zolotov, Yu. A.; Ionov; V. P. Zr. Anal. Khim. 1989, 44, 1045. Chem. Abstr. 1990, 112, 47893.

3. (a) Lin, I. C.; Pirio, M. U. S. Patent 4,742,010. Chem. Abstr. 1989, 110, 88600. (b) Attiyat, A. S.; Ibrahim, Y. A.; Christain, G. D. Microchem. J. 1988, 37, 114. (c) Attiyat, A. S.; Ibrahim, Y. A.; Christain, G. D. Microchem. J. 1988, 37, 122.

4. (a) Elwahy, A. H. M.; Muathen, H. A.; Aloweiny, N. A. M. ARKIVOC 2008, (xvii), 205211, and references cited therein. (b) Elwahy, A. H. M.; Abbas, A. A. Tetrahedron Lett. 2006, 47, 1303.

5. (a) Ibrahim, Y. A.; Elwahy, A. H. M.; Barsoum, B. N.; Abbas, A. A.; Khella, S. K. Talanta 1998, 47, 1199. (b) Barsoum, B. N.; Khella, S. K.; Elwahy, A. H. M.; Abbas, A. A.; Ibrahim, Y. A. Talanta 1998, 47, 1215. (c) Ibrahim, Y. A.; Barsoum, B. N.; Elwahy, A. H. M.; Khella, S. K. Supramol. Chem. 1998, 9, 5. (d) Abbas, A. A.; Elwahy, A. H. M. Synthesis 2001, 1331. (e) Ibrahim, Y. A.; Elwahy, A. H. M.; Abbas, A. A. Heteroat. Chem. 1994, 5, 321. (f) Abbas, A. A. Tetrahedron 1998, 54, 12421.

6. (a) Ryabokobylko, Yu. S.; Ostrovskaya, V. M.; D’yakonova, I. A.; Filatova, M. P.; Shmelev, L. V.; Kessenikh, A. V.; Fomina, L. I. Zh. Org. Chem. 1989, 25, 1759. Chem. Abstr. 1990, 112, 177868. (b) Ostrovskaya, V. M.; D’yakonova, I. A.; Poponova, R. V.; Kozlova, N. P.; Ryabokobylko, Yu. S.; Filatova, M. P. Zr. Org. Khim. 1989, 25, 1753; Chem. Abstr. 1990, 112, 178929. 